\title{
On the overall response of elastomeric solids with pressurized cavities
}

\author{
Martín I. Idiart ${ }^{\mathrm{a}, \mathrm{b}, *}$, Oscar Lopez-Pamies ${ }^{\mathrm{c}}$ \\ a Departamento de Aeronáutica, Facultad de Ingeniería, Universidad Nacional de La Plata, Avda. 1 esq. 47, La Plata B1900TAG, Argentina \\ b Consejo Nacional de Investigaciones Científicas y Técnicas (CONICET), CCT La Plata, Calle 8 No. 1467, La Plata B1904CMC, Argentina \\ c Department of Civil and Environmental Engineering, University of Illinois at Urbana-Champaign, Urbana, IL 61801-2352, USA
}

\section{A R T I C L E I N F O}

\section{Article history:}

Available online 30 March 2012

\section{Keywords:}

Elastomers

Porosity

Pressure

Residual stresses

\begin{abstract}
A B S T R A C T
This work provides a means of accounting for the presence of pressurized cavities on the overall response of elastomeric solids undergoing large deformations. The main idea is to refer the kinematics to a stress-free configuration and to express the overall response of the elastomer with pressurized cavities in terms of its overall response when the cavities are vacuous. This is achieved via a change of variables valid whenever the common assumption of incompressibility is used for the elastomeric matrix. The result permits then to incorporate straightforwardly the effect of internal pressure on any micromechanical model already available for elastomeric solids with vacuous cavities. The resulting models account for constitutive and geometric nonlinearities as well as for deformation-dependent internal pressure concomitant with large deformations. Sample results for isotropic porous rubbers under plane-strain conditions are provided and discussed.
\end{abstract}

(C) 2012 Published by Elsevier Masson SAS on behalf of Académie des sciences.

\section{Introduction}

Several micromechanical models have been proposed in recent years to account for the presence of microcavities on the mechanical response of elastomeric solids undergoing large deformations. These include models based on approximate solutions of a hollow shell [1,2], homogenization estimates based on the concept of a linear-comparison medium [3,4], and iterated-dilute homogenization estimates based on sequential microgeometries [5]. In all these models the cavities are assumed to be vacuous. There are circumstances, however, where the cavities are filled up by a gaseous phase that exerts an internal pressure on the surrounding elastomeric matrix. This internal pressure can produce, for instance, the cavitation of elastomeric seals in high-pressure gas tanks upon rapid decompression [6-8] and the shrinkage of elastomeric foams after demolding [9]. Pressurized cavities have also been recently proposed as a means to probe the mechanical properties of soft materials [10].

In order to account for the simultaneous action of internal pressure and external loading, the overall response of elastomeric solids with pressurized cavities must be determined. Inspired by recent works on the viscoplasticity of nuclear fuels $[11,12]$, we make use here of a change of variables in the relevant elastostatics problem to express the overall response of an elastomer with pressurized cavities in terms of its overall response when the cavities are vacuous. As explained in detail below, this is straightforward whenever the common assumption of incompressibility is used for the elastomeric matrix. The result - given in Section 3.3 - permits then to incorporate readily the effect of internal pressure on any micromechanical model already available for elastomeric solids with vacuous cavities. The resulting models account for constitutive and geometric nonlinearities as well as for the deformation-dependent internal pressure concomitant with large deformations.

\footnotetext{
* Corresponding author at: Departamento de Aeronáutica, Facultad de Ingeniería, Universidad Nacional de La Plata, Avda. 1 esq. 47, La Plata B1900TAG, Argentina.

E-mail addresses: martin.idiart@ing.unlp.edu.ar (M.I. Idiart), pamies@illinois.edu (O. Lopez-Pamies).
} 


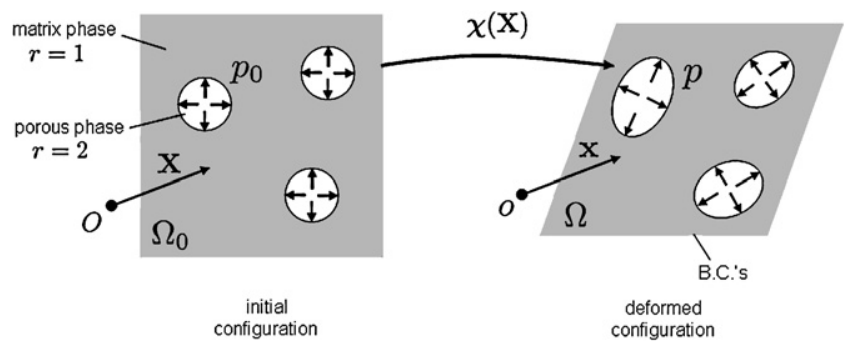

Fig. 1. Schematic of a representative volume element in its initial and deformed configurations.

\section{Local description of the material and its configurations}

\subsection{Initial and deformed configurations}

Pressurized porous elastomers are taken here to consist of a continuous incompressible matrix containing a random distribution of disconnected cavities filled with a gaseous substance. Attention is restricted to short enough time scales for which there is no (significant) diffusion of the gas into the elastomeric matrix; in other words, the gas is assumed to remain within the cavities for any given loading process.

We consider a representative volume element of the material occupying a domain $\Omega_{0}$ with boundary $\partial \Omega_{0}$ in its initial configuration. The matrix is labeled as phase $r=1$ while the filled cavities are collectively identified as phase $r=2$. The domains occupied by each individual phase are denoted by $\Omega_{0}^{(r)}$ so that $\Omega_{0}=\Omega_{0}^{(1)} \cup \Omega_{0}^{(2)}$. Consistent with the notion of representative volume element, it is assumed that the characteristic size of the cavities is much smaller than the size of $\Omega_{0}$ (this is the so-called separation-of-length-scales hypothesis), and that their random distribution in $\Omega_{0}$ is statistically uniform and ergodic.

Material points are identified by their position vector $\mathbf{X}$ in $\Omega_{0}$ relative to some fixed point $O$. The distribution of cavities can be described by an indicator function $\theta_{0}(\mathbf{X})$ that takes the value 1 if the position vector $\mathbf{X}$ is in a cavity, and 0 otherwise. The volume average of $\theta_{0}$ over $\Omega_{0}$ corresponds to the initial volume fraction of cavities, or initial porosity, which we denote by

$$
f_{0} \doteq \frac{\left|\Omega_{0}^{(2)}\right|}{\left|\Omega_{0}\right|}=\frac{1}{\left|\Omega_{0}\right|} \int_{\Omega_{0}} \theta_{0}(\mathbf{X}) \mathrm{d} \mathbf{X}
$$

Upon deformation of the solid, the position vector of a point in the deformed configuration $\Omega$ is specified by

$$
\mathbf{x}=\chi(\mathbf{X})
$$

relative to some fixed point 0 , where $\chi$ is a one-to-one mapping from $\Omega_{0}$ to $\Omega$. We assume that $\chi$ is twice continuously differentiable, except possibly on the cavities/matrix boundaries. The pointwise deformation of the matrix material will be measured by the deformation gradient tensor

$$
\mathbf{F}(\mathbf{X})=\operatorname{Grad} \chi(\mathbf{X})
$$

which must satisfy the incompressibility constraint

$$
J \doteq \operatorname{det} \mathbf{F}=1
$$

in $\Omega_{0}^{(1)}$. The initial and deformed configurations are shown schematically in Fig. 1 .

\subsection{The reference configuration}

As will become apparent further below, the mechanical response of the pressurized solid is most conveniently characterized in a reference configuration identified with the domain $\Omega_{R}$ occupied by the solid in its drained state - i.e., in the absence of gas within the cavities. Such a configuration is stress-free and corresponds to the natural state of the matrix material.

The total deformation of the solid can then be decomposed into two separate deformations, as shown schematically in Fig. 2. The position vectors $\mathbf{X}$ in the initial configuration are expressed in terms of the position vectors in the reference configuration $\mathbf{X}_{R}$ as

$$
\mathbf{X}=\chi^{\prime}\left(\mathbf{X}_{R}\right)
$$

where the function $\chi^{\prime}$ is a one-to-one mapping from $\Omega_{R}$ to $\Omega_{0}$. The corresponding deformation gradient tensor is denoted by 


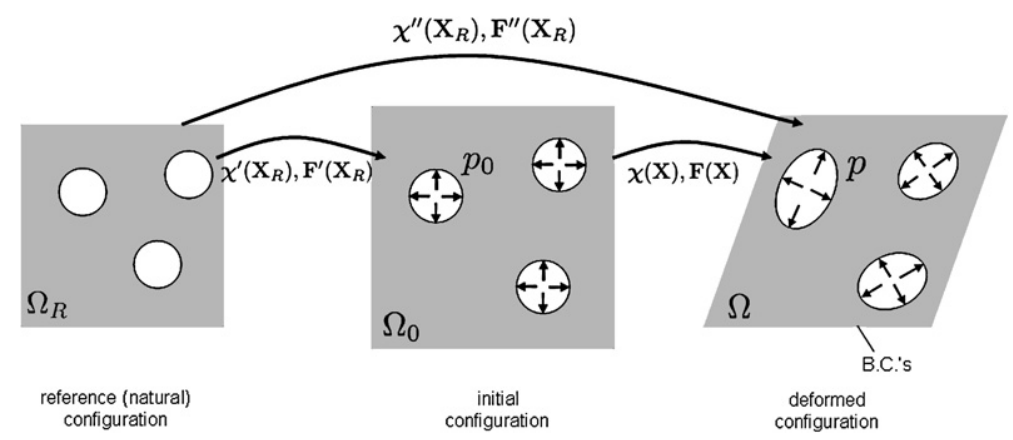

Fig. 2. Schematic of a representative volume element and its configurations.

$$
\mathbf{F}^{\prime}\left(\mathbf{X}_{R}\right)=\operatorname{Grad} \chi^{\prime}\left(\mathbf{X}_{R}\right)
$$

subject to the incompressibility constraint $J^{\prime} \doteq \operatorname{det} \mathbf{F}^{\prime}=1$ in $\Omega_{R}^{(1)}$. It is emphasized that the distribution of cavities in the reference configuration is not the same as that of the initial configuration, and should be described by an indicator function $\theta_{R}\left(\mathbf{X}_{R}\right)$ that depends on $\theta_{0}(\mathbf{X})$ and on the deformation $\chi^{\prime}$.

The position vectors $\mathbf{x}$ in the deformed configuration can be similarly expressed in terms of the position vectors $\mathbf{X}_{R}$ as

$$
\mathbf{x}=\chi^{\prime \prime}\left(\mathbf{X}_{R}\right)
$$

where $\chi^{\prime \prime}$ is a one-to-one mapping from $\Omega_{R}$ to $\Omega$. The corresponding deformation gradient tensor is denoted by

$$
\mathbf{F}^{\prime \prime}\left(\mathbf{X}_{R}\right)=\operatorname{Grad} \chi^{\prime \prime}\left(\mathbf{X}_{R}\right)
$$

subject to the incompressibility constraint $J^{\prime \prime} \doteq \operatorname{det} \mathbf{F}^{\prime \prime}=1$ in $\Omega_{R}^{(1)}$. Note that the deformation gradients (3), (6), and (8) are related via

$$
\mathbf{F}^{\prime \prime}=\mathbf{F F}^{\prime}
$$

a relation that will be invoked later in the analysis.

\subsection{Constitutive behavior of the elastomeric matrix and the gas within the cavities}

In the interest of relative simplicity, we assume here that the elastomeric matrix is incompressible and hyperelastic. Now, as a result of the internal pressure, the matrix material in the initial configuration $\Omega_{0}$ is pre-stressed. Therefore, its mechanical constitutive response is most conveniently described relative to the reference configuration $\Omega_{R}$ introduced above, where the matrix material is present in its natural state. Relative to this configuration, the local stress-deformation relations for the matrix material in the initial and deformed states take the conventional form

$$
\mathbf{S}^{\prime}=\frac{\partial W}{\partial \mathbf{F}}\left(\mathbf{F}^{\prime}\right)-q^{\prime} \mathbf{F}^{-T} \text { and } \mathbf{S}^{\prime \prime}=\frac{\partial W}{\partial \mathbf{F}}\left(\mathbf{F}^{\prime \prime}\right)-q^{\prime \prime} \mathbf{F}^{\prime \prime}-T
$$

where $W$ stands for the stored-energy function of the matrix, $\mathbf{S}^{\prime}$ and $\mathbf{S}^{\prime \prime}$ denote first Piola-Kirchhoff stress measures, and $q^{\prime}$ and $q^{\prime \prime}$ are Lagrange multipliers associated with the incompressibility constraints on $\mathbf{F}^{\prime}$ and $\mathbf{F}^{\prime \prime}$, respectively. The storedenergy function $W$ is taken here to be objective and strongly elliptic.

In view of relation $(10)_{2}$, the Cauchy stress $\mathbf{T}$ at each point $\mathbf{x} \in \Omega^{(1)}$ can be written as

$$
\mathbf{T}=\mathbf{S}^{\prime \prime} \mathbf{F}^{\prime \prime T}=\left[\frac{\partial W}{\partial \mathbf{F}}\left(\mathbf{F} \mathbf{F}^{\prime}\right)\right] \mathbf{F}^{\prime T} \mathbf{F}-q^{\prime \prime} \mathbf{I}
$$

where use has been made of relation (9). Making use of this last expression, we can define the first Piola-Kirchhoff stress $\mathbf{S}$ at each point $\mathbf{X} \in \Omega_{0}^{(1)}$ as

$$
\mathbf{S} \doteq \mathbf{T F}^{-T}=\left[\frac{\partial W}{\partial \mathbf{F}}\left(\mathbf{F F}^{\prime}\right)\right] \mathbf{F}^{\prime T} \mathbf{F F}^{-T}-q^{\prime \prime} \mathbf{F}^{-T}
$$

which provides the constitutive relation of the pre-stressed matrix material relative to the initial configuration.

In turn, we assume that the Cauchy internal pressure $p$ exerted by the gas on the matrix surrounding the cavities depends only on the current gas density $\rho$, and hence write

$$
p=\mathcal{P}(\rho)
$$


where $\mathcal{P}$ is a monotonically increasing function of $\rho$ such that $\mathcal{P} \rightarrow 0$ as $\rho \rightarrow 0$. Motivated by physical considerations (see, e.g., $[13,7])$, the initial gas density $\rho_{0}$ is assumed here to be the same in all cavities; we denote the corresponding pressure level in the initial configuration by $p_{0}=\mathcal{P}\left(\rho_{0}\right)$. For reasons that will become apparent below, it is convenient to define the Cauchy stress tensor $\mathbf{T}$ at all points $\mathbf{x} \in \Omega^{(2)}-$ and not just on $\partial \Omega^{(2)}-$ as

$$
\mathbf{T}=-p \mathbf{I}
$$

and the nominal pressure at all points $\mathbf{X} \in \Omega_{0}^{(2)}$ as

$$
\mathbf{S}=-p J \mathbf{F}^{-T}
$$

Similarly,

$$
\mathbf{S}^{\prime}=-p_{0} J^{\prime} \mathbf{F}^{\prime-T} \text { and } \mathbf{S}^{\prime \prime}=-p J^{\prime \prime} \mathbf{F}^{\prime \prime-T}
$$

denote the nominal pressures associated with the deformations $\chi^{\prime}$ and $\chi^{\prime \prime}$ at each point $\mathbf{X}_{R} \in \Omega_{R}^{(2)}$.

\section{The overall response}

Granted the hypothesis of separation of length scales and statistical uniformity in the representative volume element $\Omega_{0}$, the overall response of the pressurized solid can be defined as the relation between the volume averages of the first PiolaKirchoff stress $\mathbf{S}$ and the deformation gradient $\mathbf{F}$ over the volume $\Omega_{0}$ [14]. ${ }^{1}$ Given that the overall response is independent of the particular boundary conditions imposed on the representative volume element, we follow common practice and impose an affine boundary condition of the form

$$
\chi=\overline{\mathbf{F}} \mathbf{X} \text { on } \partial \Omega_{0}
$$

where the second-order tensor $\overline{\mathbf{F}}$ is a prescribed quantity. The average deformation gradient over $\Omega_{0}$ is then given by $\left|\Omega_{0}\right|^{-1} \int_{\Omega_{0}} \mathbf{F}(\mathbf{X}) \mathrm{d} \mathbf{X}=\overline{\mathbf{F}}$, and the derivation of the overall response reduces to finding the average stress $\overline{\mathbf{S}} \doteq$ $\left|\Omega_{0}\right|^{-1} \int_{\Omega_{0}} \mathbf{S}(\mathbf{X}) \mathrm{d} \mathbf{X}$ for a given $\overline{\mathbf{F}}$, namely, the relation

$$
\overline{\mathbf{S}}=\mathcal{S}\left(\overline{\mathbf{F}} ; \theta_{0}\right)
$$

where the dependence on the microstructure has been made explicit for clarity. Note that this function also depends on the internal pressure.

Following a similar reasoning, overall response functions relative to the reference configuration can be defined as the relations between the volume averages over the volume $\Omega_{R}$ of the first Piola-Kirchoff stress and the deformation gradient of the initial and deformed configurations. We denote those averages by $\overline{\mathbf{S}}^{\prime} \doteq\left|\Omega_{R}\right|^{-1} \int_{\Omega_{R}} \mathbf{S}^{\prime}\left(\mathbf{X}_{R}\right) \mathrm{d} \mathbf{X}_{R}$ and $\overline{\mathbf{F}}^{\prime}=\left|\Omega_{R}\right|^{-1} \int_{\Omega_{R}} \mathbf{F}^{\prime}\left(\mathbf{X}_{R}\right) \mathrm{d} \mathbf{X}_{R}$, and similarly by $\overline{\mathbf{S}}^{\prime \prime} \doteq\left|\Omega_{R}\right|^{-1} \int_{\Omega_{R}} \mathbf{S}^{\prime \prime}\left(\mathbf{X}_{R}\right) \mathrm{d} \mathbf{X}_{R}$ and $\overline{\mathbf{F}}^{\prime \prime}=\left|\Omega_{R}\right|^{-1} \int_{\Omega_{R}} \mathbf{F}^{\prime \prime}\left(\mathbf{X}_{R}\right) \mathrm{d} \mathbf{X}_{R}$, and write their relations as

$$
\overline{\mathbf{S}}^{\prime}=\mathcal{S}^{\prime}\left(\overline{\mathbf{F}}^{\prime} ; \theta_{R}\right) \quad \text { and } \quad \overline{\mathbf{S}}^{\prime \prime}=\mathcal{S}^{\prime \prime}\left(\overline{\mathbf{F}}^{\prime \prime} ; \theta_{R}\right)
$$

Like the function $\mathcal{S}$, these functions depend on the internal pressure but, unlike the function $\mathcal{S}$, are referred to a stress-free natural configuration.

Now, the function $\mathcal{S}$ can be written in terms of $\mathcal{S}^{\prime \prime}$ by making use of the fact that the overall Cauchy stress in the deformed configuration can be expressed as

$$
\overline{\mathbf{T}}=\frac{1}{\bar{J}^{\prime \prime}} \overline{\mathbf{S}}^{\prime \prime} \overline{\mathbf{F}}^{\prime \prime T}=\frac{1}{\bar{J}} \overline{\mathbf{S}} \overline{\mathbf{F}}^{T}
$$

where $\overline{\mathbf{T}} \doteq|\Omega|^{-1} \int_{\Omega} \mathbf{T}(\mathbf{x}) \mathrm{d} \mathbf{x}, \bar{J} \doteq \operatorname{det} \overline{\mathbf{F}}$, and $\bar{J}^{\prime \prime} \doteq \operatorname{det} \overline{\mathbf{F}}^{\prime \prime}$. The validity of this relation rests upon the assumed separation of length scales whereby the porous solid is regarded macroscopically as a homogeneous material. From this relation we deduce that

$$
\mathcal{S}\left(\overline{\mathbf{F}} ; \theta_{0}\right)=\frac{\bar{J}}{\bar{J}^{\prime \prime}} \mathcal{S}^{\prime \prime}\left(\overline{\mathbf{F}}^{\prime \prime} ; \theta_{0}^{\prime}\right) \overline{\mathbf{F}}^{\prime \prime T} \overline{\mathbf{F}}^{-T}
$$

In turn, similar to their local counterpart (9), the overall deformation gradients $\overline{\mathbf{F}}$ and $\overline{\mathbf{F}}^{\prime \prime}$ are related by

$$
\overline{\mathbf{F}}^{\prime \prime}=\overline{\mathbf{F}} \overline{\mathbf{F}}^{\prime}
$$

\footnotetext{
1 See also $[15,16]$ for more mathematically precise definitions in the context of hyperelastic solids with periodic microstructures
} 
where $\overline{\mathbf{F}}^{\prime}=\left|\Omega_{R}\right|^{-1} \int_{\Omega_{R}} \mathbf{F}^{\prime}\left(\mathbf{X}_{R}\right) \mathrm{d} \mathbf{X}_{R}$. Given that the initial configuration is traction-free on its external boundary, the tensor $\overline{\mathbf{F}}^{\prime}$ is solution to the equation

$$
\mathcal{S}^{\prime}\left(\overline{\mathbf{F}}^{\prime} ; \theta_{R}\right)=\mathbf{0}
$$

Comments on the possibility of multiple solutions to the algebraic nonlinear equation (23) are deferred to Section 3.3 .

Once $\overline{\mathbf{F}}^{\prime}$ is obtained from Eq. (23), the overall response (21) can be finally written as

$$
\mathcal{S}\left(\overline{\mathbf{F}} ; \theta_{0}\right)=\frac{1}{\bar{J}^{\prime}} \mathcal{S}^{\prime \prime}\left(\overline{\mathbf{F}} \overline{\mathbf{F}}^{\prime} ; \theta_{0}^{\prime}\right) \overline{\mathbf{F}}^{\prime T}
$$

where $\bar{J}^{\prime}=\operatorname{det} \overline{\mathbf{F}}^{\prime}$. Thus, the computation of $\mathcal{S}$ reduces to finding the overall response functions $\mathcal{S}^{\prime}$ and $\mathcal{S}^{\prime \prime}$, which are referred to a natural configuration with microstructure $\theta_{R}$. Now, these functions still depend on the internal pressure in view of expressions (16) for the stresses in the pressurized cavities. However, they can be expressed in terms of the response function of the drained solid making use of a suitable change of variables, as shown next.

\subsection{Overall response function $\mathcal{S}^{\prime}$}

The tensor function $\mathcal{S}^{\prime}$ represents the relation between the volume averages of the fields $\mathbf{F}^{\prime}\left(\mathbf{X}_{R}\right)$ and $\mathbf{S}^{\prime}\left(\mathbf{X}_{R}\right)$ over the reference configuration $\Omega_{R}$. These fields are solution to the following boundary-value problem:

$$
\begin{array}{ll}
\operatorname{Div} \mathbf{S}^{\prime}=\mathbf{0} & \text { in } \Omega_{R} \\
\mathbf{S}^{\prime}=\frac{\partial W}{\partial \mathbf{F}}\left(\mathbf{F}^{\prime}\right)-q^{\prime} \mathbf{F}^{\prime}-T & \text { in } \Omega_{R}^{(1)} \\
\mathbf{S}^{\prime}=-p_{0} J^{\prime} \mathbf{F}^{\prime}-T & \text { in } \Omega_{R}^{(2)} \\
\mathbf{F}^{\prime}=\operatorname{Grad} \chi^{\prime} & \text { in } \Omega_{R} \\
J^{\prime}=1 & \text { in } \Omega_{R}^{(1)} \\
\mathbf{S}^{\prime} \mathbf{N}=\mathbf{0} & \text { on } \partial \Omega_{R}
\end{array}
$$

where $p_{0}$ is a prescribed quantity. Here, it is important to emphasize that the mechanical fields $\chi^{\prime}$ and $\mathbf{S}^{\prime}$ are defined over the entire domain $\Omega_{R}$ - and not just over the matrix material $\Omega_{R}^{(1)}$ - by making use of the description (14)-(16) for the gas within the cavities and the fact that $\operatorname{Div}\left[J^{\prime} \mathbf{F}^{\prime}-T=\mathbf{0}\right.$.

Now, borrowing ideas from the works of Julien et al. [11] and Vincent et al. [12] on viscoplastic nuclear fuels, we rewrite the above problem as a boundary-value problem for a drained solid with the same microstructure $\theta_{R}$. This is achieved by introducing the change of variables

$$
\hat{\mathbf{S}}^{\prime} \doteq \mathbf{S}^{\prime}+p_{0} J^{\prime} \mathbf{F}^{\prime-T}
$$

so that, in view of the assumed uniformity of $p_{0}$ and the fact that the field $J^{\prime} \mathbf{F}^{\prime-T}$ is divergence-free, Eqs. (25) take the form

$$
\begin{array}{ll}
\operatorname{Div} \hat{\mathbf{S}}^{\prime}=\mathbf{0} & \text { in } \Omega_{R} \\
\hat{\mathbf{S}}^{\prime}=\frac{\partial W}{\partial \mathbf{F}}\left(\mathbf{F}^{\prime}\right)-\hat{q}^{\prime} \mathbf{F}^{\prime}-T & \text { in } \Omega_{R}^{(1)} \\
\hat{\mathbf{S}}^{\prime}=\mathbf{0} & \text { in } \Omega_{R}^{(2)} \\
\mathbf{F}^{\prime}=\operatorname{Grad} \chi^{\prime} & \text { in } \Omega_{R} \\
J^{\prime}=1 & \text { in } \Omega_{R}^{(1)} \\
\chi^{\prime}=\overline{\mathbf{F}}^{\prime} \mathbf{X} & \text { on } \partial \Omega_{R}
\end{array}
$$

where, for convenience, we have also replaced the boundary condition on the stress $(25)_{6}$ for the equivalent affine boundary condition on the deformation $(27)_{6}$ with $\overline{\mathbf{F}}^{\prime}$ solution to (23).

At this point, it is a simple matter to recognize from condition $(27)_{3}$ that the stress field $\hat{\mathbf{S}}^{\prime}$ corresponds to that of a drained solid, and therefore, the relation between the volume averages of the fields $\mathbf{F}^{\prime}$ and $\hat{\mathbf{S}}^{\prime}$ is given by the overall response $\tilde{\mathcal{S}}$ of a drained solid relative to its natural state. By averaging relation (26) over $\Omega_{R}$ we arrive at the following expression for $\mathcal{S}^{\prime}$ in terms of $\tilde{\mathcal{S}}$ :

$$
\mathcal{S}^{\prime}\left(\overline{\mathbf{F}}^{\prime} ; \theta_{R}\right)=\tilde{\mathcal{S}}\left(\overline{\mathbf{F}}^{\prime} ; \theta_{R}\right)-p_{0} \bar{J}^{\prime} \overline{\mathbf{F}}^{\prime-T}
$$

where use has been made of the identity $\left|\Omega_{R}\right|^{-1} \int_{\Omega_{R}} J^{\prime} \mathbf{F}^{\prime-T} \mathrm{~d} \mathbf{X}_{R}=\bar{J}^{\prime} \overline{\mathbf{F}}^{\prime-T}$. 


\subsection{Overall response function $\mathcal{S}^{\prime \prime}$}

The tensor function $\mathcal{S}^{\prime \prime}$ represents the relation between the volume averages of the fields $\mathbf{F}^{\prime \prime}\left(\mathbf{X}_{R}\right)$ and $\mathbf{S}^{\prime \prime}\left(\mathbf{X}_{R}\right)$ over the reference configuration $\Omega_{R}$. Similar to the preceding case, these fields are solution to the following boundary value problem:

$$
\begin{array}{ll}
\operatorname{Div} \mathbf{S}^{\prime \prime}=\mathbf{0} & \text { in } \Omega_{R} \\
\mathbf{S}^{\prime \prime}=\frac{\partial W}{\partial \mathbf{F}}\left(\mathbf{F}^{\prime \prime}\right)-q^{\prime \prime} \mathbf{F}^{\prime \prime}-T & \text { in } \Omega_{R}^{(1)} \\
\mathbf{S}^{\prime \prime}=-p J^{\prime \prime} \mathbf{F}^{\prime \prime-T} & \text { in } \Omega_{R}^{(2)} \\
\mathbf{F}^{\prime \prime}=\operatorname{Grad} \boldsymbol{\chi}^{\prime \prime} & \text { in } \Omega_{R} \\
J^{\prime \prime}=1 & \text { in } \Omega_{R}^{(1)} \\
\boldsymbol{\chi}^{\prime \prime}=\overline{\mathbf{F}}^{\prime \prime} \mathbf{X}_{R} & \text { on } \partial \Omega_{R}
\end{array}
$$

where the second-order tensor $\overline{\mathbf{F}}^{\prime \prime}$ is a prescribed quantity and the internal pressure $p$ is given by (13). Now, since each cavity will grow differently during a general deformation process, the pressure $p$ will take different values in different cavities. This prevents the use of the change of variables introduced in the previous section to express the response function $\mathcal{S}^{\prime \prime}$ in terms of the function $\tilde{\mathcal{S}}$. However, noting that most of the available models for porous elastomers assume that all cavities undergo the same deformation, the internal pressure $p$ is taken here to have the same value in all cavities throughout the deformation process. Granted this uniformity of the internal pressure and matrix incompressibility, we can then write

$$
p=\mathcal{P}(\rho)=\mathcal{P}\left(\frac{\rho_{0} f_{0}}{\bar{J}-1+f_{0}}\right) \doteq \overline{\mathcal{P}}(\bar{J})
$$

so that the local pressure $p$ in all the cavities can also be regarded as a prescribed quantity for a given macroscopic deformation $\overline{\mathbf{F}}$. The above problem can then be rewritten as a boundary-value problem for a drained solid by following the strategy of the previous section, and the overall response function $\mathcal{S}^{\prime \prime}$ can be expressed in terms of $\tilde{\mathcal{S}}$ as

$$
\mathcal{S}^{\prime \prime}\left(\overline{\mathbf{F}}^{\prime \prime} ; \theta_{R}\right)=\tilde{\mathcal{S}}\left(\overline{\mathbf{F}}^{\prime \prime} ; \theta_{R}\right)-\overline{\mathcal{P}}(\bar{J}) \bar{J}^{\prime \prime} \overline{\mathbf{F}}^{\prime \prime}-T
$$

\subsection{Overall response function $\mathcal{S}$ of the pressurized solid}

Combining (24) and (31) finally renders

$$
\mathcal{S}\left(\overline{\mathbf{F}} ; \theta_{0}\right)=\frac{1}{\bar{J}^{\prime}} \tilde{\mathcal{S}}\left(\overline{\mathbf{F}} \overline{\mathbf{F}}^{\prime} ; \theta_{R}\right) \overline{\mathbf{F}}^{\prime T}-\overline{\mathcal{P}}(\bar{J}) \bar{J} \overline{\mathbf{F}}^{-T}
$$

where $\overline{\mathbf{F}}^{\prime}$ is a solution to

$$
\tilde{\mathcal{S}}\left(\overline{\mathbf{F}}^{\prime} ; \theta_{R}\right)=p_{0} \bar{J}^{\prime} \overline{\mathbf{F}}^{\prime-T}
$$

in view of expressions (23) and (28). Relation (32) constitutes the main result of this paper: it expresses the overall response function $\mathcal{S}$ of the solid containing pressurized cavities in terms of the initial pressure within the cavities and the overall response function $\tilde{\mathcal{S}}$ of a drained solid with vacuous cavities.

For completeness, we recall here that the overall response of the drained solid can be written as [14]

$$
\tilde{\mathcal{S}}\left(\overline{\mathbf{F}} ; \theta_{R}\right)=\frac{\partial \tilde{\mathcal{W}}}{\partial \overline{\mathbf{F}}}\left(\overline{\mathbf{F}} ; \theta_{R}\right)
$$

where $\tilde{\mathcal{W}}$ is the so-called effective stored-energy function defined by

$$
\tilde{\mathcal{W}}\left(\overline{\mathbf{F}} ; \theta_{R}\right)=\min _{\mathbf{F}^{\prime \prime} \in \mathcal{K}(\overline{\mathbf{F}})} \frac{1}{\left|\Omega_{R}\right|} \int_{\Omega_{R}}\left[1-\theta_{R}\left(\mathbf{X}_{R}\right)\right] W\left(\mathbf{F}^{\prime \prime}\right) \mathrm{d} \mathbf{X}_{R}
$$

In this last relation, $\mathcal{K}$ denotes a suitable set of kinematically admissible deformation gradients $\mathbf{F}^{\prime \prime}$ with prescribed volume average $\left|\Omega_{R}\right|^{-1} \int_{\Omega_{R}} \mathbf{F}^{\prime \prime}\left(\mathbf{X}_{R}\right) \mathrm{d} \mathbf{X}_{R}=\overline{\mathbf{F}}$; see, for instance, [17,18] and references therein. Physically, the function $\tilde{\mathcal{W}}$ represents the total elastic energy stored in the solid.

In view of (34), it is interesting to recognize that the constitutive relation (32) can be rewritten as

$$
\mathcal{S}\left(\overline{\mathbf{F}} ; \theta_{0}\right)=\frac{\partial \mathcal{W}}{\partial \overline{\mathbf{F}}}\left(\overline{\mathbf{F}} ; \theta_{0}\right)
$$

where $\mathcal{W}$ can be thought of as the effective stored-energy function of the pressurized elastomer and is defined as 


$$
\mathcal{W}\left(\overline{\mathbf{F}} ; \theta_{0}\right)=\frac{1}{\bar{J}^{\prime}} \tilde{\mathcal{W}}\left(\overline{\mathbf{F}} \overline{\mathbf{F}}^{\prime} ; \theta_{R}\right)+\int_{\bar{J}}^{\bar{J}_{0}} \overline{\mathcal{P}}(z) \mathrm{d} z
$$

where $\bar{J}_{0}$ is an arbitrary positive constant. Note that for $\overline{\mathbf{F}}=\mathbf{I}$ this function is in general different from zero.

A few comments regarding the above formulae are in order. Firstly, the reference microstructure $\theta_{R}$ will depend on $\overline{\mathbf{F}}^{\prime}$ in an intricate manner and, consequently, solving Eq. (33) will be complicated in general. However, homogenization estimates do not require complete knowledge of the microstructure, and if the initial porosity and the matrix material in its natural state exhibit certain symmetries, significant simplifications can be expected (see Section 4 below for an example). Secondly, owing to its nonlinearity, the algebraic equation (33) may have multiple solutions, but given that the solution $\overline{\mathbf{F}}^{\prime}$ represents the overall deformation gradient of the initial configuration relative to the natural configuration of the drained solid, and that the microstructure of the initial configuration is prescribed, only one solution is expected to be admissible. Note also that primed quantities depend nonlinearly on the initial level of internal pressure, and so the overall response of the pressurized solid depends on internal pressure in a highly nontrivial manner.

\subsection{A remark on the macroscopic stability of the pressurized solid}

In addition to characterizing the macroscopic constitutive response, the above formalism can provide information about the onset of macroscopic instabilities, that is, geometric instabilities with wavelengths much larger than the characteristic size of the microstructure $[19,20]$. During an arbitrary loading path starting from the initial configuration, the pressurized solid may become macroscopically unstable whenever the condition

$$
\min _{\|\mathbf{u}\|=\|\mathbf{v}\|=1} Q\left(\mathbf{u}, \mathbf{v}, \overline{\mathbf{F}} ; \theta_{0}\right)=0
$$

is first satisfied; here,

$$
Q\left(\mathbf{u}, \mathbf{v}, \overline{\mathbf{F}} ; \theta_{0}\right)=\mathcal{L}_{i j k l}\left(\overline{\mathbf{F}} ; \theta_{0}\right) u_{j} u_{l} v_{i} v_{k}
$$

where

$$
\mathcal{L}_{i j k l}\left(\overline{\mathbf{F}} ; \theta_{0}\right)=\bar{F}_{j p} \bar{F}_{l q} \frac{\partial \mathcal{S}_{k q}}{\partial \bar{F}_{i p}}\left(\overline{\mathbf{F}} ; \theta_{0}\right)
$$

is the overall instantaneous modulus tensor of the pressurized solid [21].

Making use of (32), the function $Q$ for the pressurized solid can be rewritten as

$$
Q\left(\mathbf{u}, \mathbf{v}, \overline{\mathbf{F}} ; \theta_{0}\right)=\frac{1}{\bar{J}^{\prime}} \tilde{Q}\left(\mathbf{u}, \mathbf{v}, \overline{\mathbf{F}} \overline{\mathbf{F}}^{\prime} ; \theta_{R}\right)-\bar{J}^{2} \frac{\mathrm{d} \overline{\mathcal{P}}}{\mathrm{d} \bar{J}}(\bar{J})(\mathbf{u} \cdot \mathbf{v})^{2}
$$

where $\tilde{Q}$ is defined in terms of the response function $\tilde{\mathcal{S}}$ by an expression analogous to (39). Note that $\overline{\mathcal{P}}(\bar{J})$ is a decreasing function of $\bar{J}$, since gas pressure should decrease with increasing volume. The second term inside the square brackets in (41) is thus nonnegative, suggesting that internal pressure increases the overall macroscopic stability of the solid. In particular, the above conditions imply that if the drained solid with microstructure $\theta_{R}$ is strongly elliptic, so is the pressurized solid with microstructure $\theta_{0}$.

\section{Sample results for a 2D isotropic porous rubber under plane-strain conditions}

For demonstration purposes, we make use of the above formalism to compute the overall response of a model rubbery solid containing pressurized cavities. The cavities consist of a single family of aligned cylinders which are isotropically distributed in the transverse plane, and the solid is subjected to plane-strain conditions. The problem is formulated in two dimensions.

The matrix material is characterized by an incompressible Neo-Hookean energy of the form

$$
W(\mathbf{F})= \begin{cases}\frac{\mu}{2}(\mathbf{F} \cdot \mathbf{F}-2) & \text { if } \operatorname{det} \mathbf{F}=1 \\ +\infty & \text { otherwise }\end{cases}
$$

where the positive material parameter $\mu>0$ stands for the shear modulus in the ground state. In turn, the gas pressure is assumed to depend linearly on the gas density, as in for instance the ideal gas model, so that

$$
\mathcal{P}(\bar{J})=\frac{f_{0}}{\bar{J}-1+f_{0}} p_{0}
$$

Given that the matrix material and the in-plane distribution of porosity are isotropic, the solution to Eq. (33) in this case must be of the form 


$$
\overline{\mathbf{F}}^{\prime}=\sqrt{\bar{J}^{\prime}} \mathbf{I}
$$

and the porosity distribution $\theta_{R}$ in the reference configuration must be isotropic. In view of the matrix incompressibility, the porosity in the reference configuration must in turn be expressible as

$$
f_{R}=1-\bar{J}^{\prime}\left(1-f_{0}\right)
$$

A constitutive model for the drained solid is now required. By way of example we make use here of an iterated-dilute homogenization model recently proposed by Lopez-Pamies and Idiart [5]. This model incorporates one- and two-point microstructural statistics, and has the distinguishing feature of being realizable in the sense that it is exact for porous solids with a particular class of microgeometries. According to this model the effective stored-energy function (35) is, with a slight abuse of notation, given by

$$
\tilde{\mathcal{W}}\left(\overline{\mathbf{F}} ; f_{R}\right)=\frac{1}{2} \tilde{\mu}\left(f_{R}\right)(\bar{I}-2)+(\bar{J}-1)\left\{\frac{\mu}{2} \ln \left[\frac{\bar{J}-1+f_{R}}{f_{R} \bar{J}}\right]-\tilde{\mu}\left(f_{R}\right)\right\}
$$

where $\bar{I}=\overline{\mathbf{F}} \cdot \overline{\mathbf{F}}$ is the first principal invariant of $\overline{\mathbf{F}}^{T} \overline{\mathbf{F}}$, and

$$
\tilde{\mu}\left(f_{R}\right)=\frac{1-f_{R}}{1+f_{R}} \mu
$$

denotes its effective shear modulus in the ground state. The corresponding overall response function is given by

$$
\tilde{\mathcal{S}}\left(\overline{\mathbf{F}} ; f_{R}\right)=\frac{\partial \tilde{\mathcal{W}}}{\partial \overline{\mathbf{F}}}\left(\overline{\mathbf{F}} ; f_{R}\right)=\tilde{\mu}\left(f_{R}\right)\left(\overline{\mathbf{F}}-\bar{J} \overline{\mathbf{F}}^{-T}\right)+\frac{\mu}{2}\left\{\bar{J} \ln \left[\frac{\bar{J}-1+f_{R}}{f_{R} \bar{J}}\right]+\frac{\left(1-f_{R}\right)(\bar{J}-1)}{\bar{J}-1+f_{R}}\right\} \overline{\mathbf{F}}^{-T}
$$

Note that the only microstructural parameter that appears explicitly in this model is the porosity $f_{R}$ in the reference configuration.

On combining the response function (48) with Eq. (33), and taking the trace of the resulting tensorial equation, we obtain the following scalar equation for $\bar{J}^{\prime}$ :

$$
\ln \left[\frac{\bar{J}^{\prime}-1+f_{R}}{f_{R} \bar{J}^{\prime}}\right]+\frac{\left(1-f_{R}\right)\left(\bar{J}^{\prime}-1\right)}{\bar{J}^{\prime}\left(\bar{J}^{\prime}-1+f_{R}\right)}=\frac{2 p_{0}}{\mu}
$$

where $f_{R}$ is given in terms of $\bar{J}^{\prime}$ by (45). At this point, by making use of (48) and (32), it is a simple matter to deduce that the overall response function of the pressurized solid is given by

$$
\mathcal{S}\left(\overline{\mathbf{F}} ; f_{0}\right)=\tilde{\mu}\left(f_{R}\right)\left(\overline{\mathbf{F}}-\bar{J} \overline{\mathbf{F}}^{-T}\right)+\frac{\mu}{2}\left\{\bar{J} \ln \left[\frac{\bar{J}^{\prime} \bar{J}-1+f_{R}}{f_{R} \bar{J}^{\prime} \bar{J}}\right]+\frac{\left(1-f_{R}\right)\left(\bar{J}^{\prime} \bar{J}-1\right)}{\bar{J}^{\prime}\left(\bar{J}^{\prime} \bar{J}-1+f_{R}\right)}-\frac{2 f_{0} \bar{J}}{\bar{J}-1+f_{0}} \frac{p_{0}}{\mu}\right\} \overline{\mathbf{F}}^{-T}
$$

Note that this expression depends very intricately on the initial internal pressure $p_{0}$ through the reference porosity $f_{R}$, as defined by relation (45), and through the variable $\bar{J}^{\prime}$, as defined by the nonlinear algebraic equation (49).

For the limiting cases of isochoric $(\bar{J}=1)$ and purely hydrostatic $(\bar{I}=2)$ loading conditions, the overall constitutive equation (50) admits some simplification worth recording. Indeed, it is easy to show that for isochoric loadings expression (50) reduces to

$$
\mathcal{S}\left(\overline{\mathbf{F}} ; f_{0}\right)=\tilde{\mu}\left(f_{R}\right)\left(\overline{\mathbf{F}}-\overline{\mathbf{F}}^{-T}\right)
$$

Note that this expression still depends on $p_{0}$ via $f_{R}$. The fact that $f_{R}<f_{0}$ and $\tilde{\mu}\left(f_{R}\right)>\tilde{\mu}\left(f_{0}\right)$ makes plain the stiffening of the material response due to the internal pressure. Under isotropic deformations of the form $\overline{\mathbf{F}}=\sqrt{\bar{J}} \mathbf{I}$, expression (50) reduces to

$$
\mathcal{S}\left(\overline{\mathbf{F}} ; f_{0}\right)=\frac{\mu}{2}\left\{\sqrt{\bar{J}} \ln \left[\frac{\bar{J}^{\prime} \bar{J}-1+f_{R}}{f_{R} \bar{J}^{\prime} \bar{J}}\right]+\frac{\left(1-f_{R}\right)\left(\bar{J}^{\prime} \bar{J}-1\right)}{\bar{J}^{\prime} \sqrt{\bar{J}}\left(\bar{J}^{\prime} \bar{J}-1+f_{R}\right)}-\frac{2 f_{0} \sqrt{\bar{J}}}{\bar{J}-1+f_{0}} \frac{p_{0}}{\mu}\right\} \mathbf{I}
$$

It is recalled that the hydrostatic response predicted by (46) agrees exactly with the hydrostatic response of a hollow shell with the same void content (see Section 2 in [5]). The predicted stress-deformation response is shown in Fig. 3 for the choice $f_{0}=0.01$ and various internal pressure levels $\left(p_{0} / \mu=0,1,5,10\right)$, with $\bar{s}$ such that $\overline{\mathbf{S}}=\bar{s} \mathbf{I}$. The strong stiffening effect due to internal pressure is evident under both extension, part (a), and compression, part (b). In fact, an asymptotic analysis of Eqs. (45) and (49) reveals that the porosity $f_{R}$ in the reference configuration decreases exponentially with increasingly large pressure $p_{0}$ as $f_{R} / f_{0}=e^{-2 p_{0} / \mu}+O(1)$. Thus, as soon as $p_{0}$ is a few times the shear modulus $\mu$ of the elastomeric matrix, the reference configuration becomes an almost fully dense solid exhibiting quasi-incompressible behavior, hence the strong stiffening observed. We conclude this analysis by noting that the stored-energy function (46) for the drained solid is strongly elliptic for all deformations (see Section 2 in [5]) and therefore the pressurized solid remains so for any level of internal pressure. 
a)

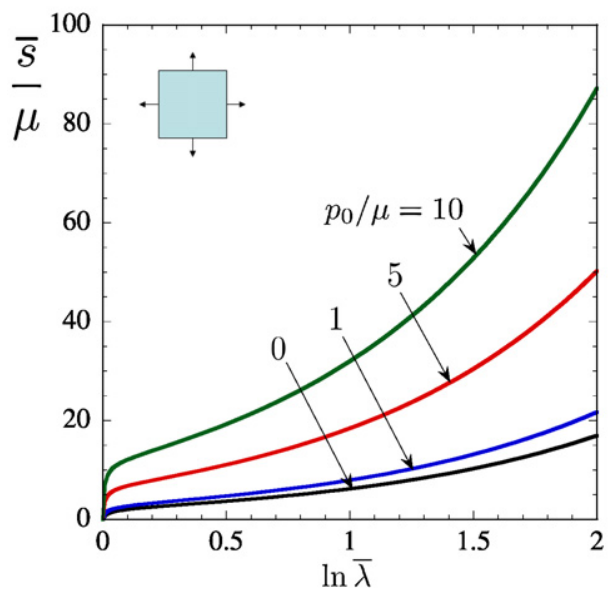

b)

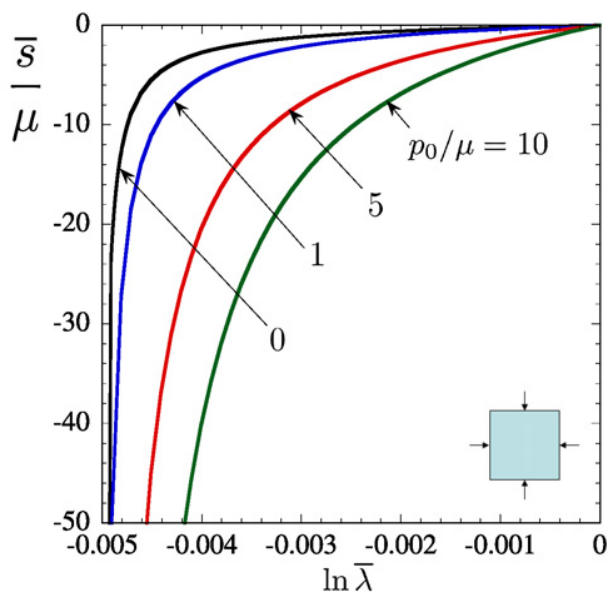

Fig. 3. Overall hydrostatic response of Neo-Hookean solids for various values of internal pressure $\left(p_{0} / \mu=0,1,5,10\right)$ : a) hydrostatic extension, b) hydrostatic compression. The initial porosity is $f_{0}=0.01$.

\section{Final comments}

The formalism derived in this work can be utilized to study a variety of problems concerning porous elastomeric systems wherein the cavities are pressurized. For instance, in the high-porosity regime, it can be used to study the mechanical response and macroscopic stability of closed-cell elastomeric foams that are operated in atmospheric and higher pressure environments. Given that the matrix materials used in such foams often have initial elastic moduli on the order of 1 atm, it is anticipated that atmospheric and higher internal pore pressures may considerably influence the response of these materials.

In the low-porosity regime, the above formalism can be used to study the failure of elastomeric seals in high-pressure gas tanks upon rapid gas decompression [6]. When a gas is dissolved in an elastomer under high external pressure and the pressure is then released, the gas comes out of solution. While part of this gas diffuses outwards through the edges of the specimen, most of it diffuses into preexisting submicrometer cavities which grow in size as a result of the internal pressure $[13,8]$. At some point after the release of external pressure, the gas contained in the cavities progressively migrates out of the specimen releasing the internal pressure. If during this process the maximum level reached by the internal pressure remains below a certain critical level, the cavities collapse back to their initial sizes [13]; above that critical level, however, permanent damage in the form of blistering or "pop-corn" failure occurs [7]. The results presented in this work can be used in conjunction with existing defect-growth theories of cavitation, such as the one recently proposed in [22,23], to determine the critical pressure for blistering under arbitrary external loadings.

Finally, the formalism may also be useful to account for cavity surface tension on the overall response of porous elastomers, given that surface tension enters the elastostatics equations in the form of an internal (Laplace) pressure. The effect of surface tension becomes important at sufficiently small cavity sizes [24], and according to recent analyses it may turn cavity growth unstable [25].

\section{Acknowledgements}

The work of M.I.I. was supported by CONICET (Argentina) through Grant PIP 00394/10. The work of O.L.P. was supported by the National Science Foundation (USA) through Grant NSF/DMS-1009503.

\section{References}

[1] M. Danielsson, D.M. Parks, M.C. Boyce, Constitutive modeling of porous hyperelastic materials, Mech. Mater. 36 (2004) $347-358$.

[2] Z. Hashin, Large isotropic elastic deformation of composites and porous media, Int. J. Solids Struct. 21 (1985) $711-720$.

[3] O. Lopez-Pamies, P. Ponte Castañeda, Homogenization-based constitutive models for porous elastomers and implications for macroscopic instabilities: I-Analysis, J. Mech. Phys. Solids 55 (2007) 1677-1701.

[4] O. Lopez-Pamies, P. Ponte Castañeda, Homogenization-based constitutive models for porous elastomers and implications for macroscopic instabilities: II-Results, J. Mech. Phys. Solids 55 (2007) 1702-1728.

[5] O. Lopez-Pamies, M.I. Idiart, An exact result for the macroscopic response of porous Neo-Hookean solids, J. Elast. 95 (2009) $99-105$.

[6] E. Ho, Elastomeric seals for rapid gas decompression applications in high-pressure services, BHR Group Limited Research Report 485 for the Health and Safety Executive 2006, HSE Books, Sudbury, UK.

[7] J. Yamabe, S. Nishimura, Influence of fillers on hydrogen penetration properties and blister fracture of rubber composites for O-ring exposed to highpressure hydrogen gas, Int. J. Hydrogen Energy 34 (2009) 1977-1989.

[8] J. Yamabe, T. Matsumoto, S. Nishimura, Application of acoustic emission method to detection of internal fracture of sealing rubber material by highpressure hydrogen decompression, Polym. Testing 30 (2011) 76-85. 
[9] T. Fen-Chong, E. Hervé, A. Zaoui, Micromechanical modelling of intracellular pressure-induced viscoelastic shrinkage of foams: application to expanded polystyrene, Eur. J. Mech. A/Solids 18 (1999) 201-218.

[10] S. Kundu, A.J. Crosby, Cavitation and fracture behavior of polyacrylamide hydrogels, Soft Matter 5 (2009) 3963-3968.

[11] J. Julien, M. Garajeu, J.-C. Michel, A semi-analytical model for the behavior of saturated viscoplastic materials containing two populations of voids of different sizes, Int. J. Solids Struct. 48 (2011) 1485-1498.

[12] P.-G. Vincent, Y. Monerie, P. Suquet, Porous materials with two populations of voids under internal pressure: I. Instantaneous constitutive relations, Int. J. Solids Struct. 46 (2009) 480-506.

[13] A.N. Gent, D.A. Tompkins, Nucleation and growth of gas bubbles in elastomers, J. Appl. Phys. 40 (1969) 2520-2525.

[14] R. Hill, On constitutive macro-variables for heterogeneous solids at finite strain, Proc. R. Soc. Lond. A 326 (1972) 131-147.

[15] A. Braides, Homogenization of some almost periodic coercive functionals, Rend. Accad. Naz. Sci. XL 9 (1985) 313-322.

[16] S. Müller, Homogenization of nonconvex integral functionals and cellular elastic materials, Arch. Ration. Mech. Anal. 99 (1987) 189-212.

[17] O. Lopez-Pamies, P. Ponte Castañeda, On the overall behavior, microstructure evolution, and macroscopic stability in reinforced rubbers at large deformations: I-Theory, J. Mech. Phys. Solids 54 (2006) 807-830.

[18] O. Lopez-Pamies, M.I. Idiart, Fiber-reinforced hyperelastic solids: a realizable homogenization constitutive theory, J. Eng. Math. 68 (2010) $57-83$.

[19] G. Geymonat, S. Müller, N. Triantafyllidis, Homogenization of nonlinearly elastic materials, microscopic bifurcation and macroscopic loss of rank-one convexity, Arch. Ration. Mech. Anal. 122 (1993) 231-290.

[20] J.C. Michel, O. Lopez-Pamies, P. Ponte Castañeda, N. Triantafyllidis, Microscopic and macroscopic instabilities in finitely strained porous elastomers, J. Mech. Phys. Solids 55 (2007) 900-938.

[21] P. Chadwick, R.W. Ogden, On the definition of elastic moduli, Arch. Ration. Mech. Anal. 44 (1971) 41-53.

[22] O. Lopez-Pamies, M.I. Idiart, T. Nakamura, Cavitation in elastomeric solids: I-A defect-growth theory, J. Mech. Phys. Solids 59 (2011) $1464-1487$.

[23] O. Lopez-Pamies, T. Nakamura, M.I. Idiart, Cavitation in elastomeric solids: II-Onset-of-cavitation surfaces for Neo-Hookean materials, J. Mech. Phys. Solids 59 (2011) 1488-1505.

[24] A.N. Gent, D.A. Tompkins, Surface effects for small holes or particles in elastomers, J. Polym. Sci. Part A 2 (7) (1969) 1483-1488.

[25] J. Zhu, T. Li, S. Cai, Z. Suo, Snap-through expansion of a gas bubble in an elastomer, J. Adhesion 87 (2011) 466-481. 